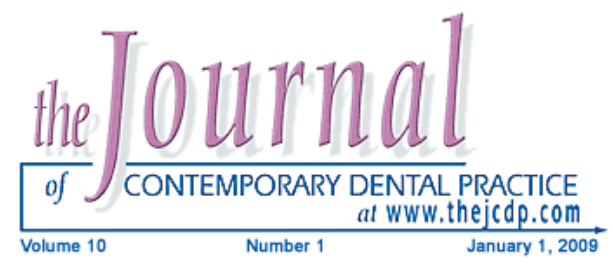

\title{
Lingual Eruption of Mandibular Permanent Incisors: A Space Correlated Phenomenon?
}

\author{
Naser AsI Aminabadi, DDS, MSc; Ramin Mostofi Zadeh Farahani, DDS; \\ Aydin Sohrabi, DDS, MSc; Firuz Pouralibaba, DDS, MSc
}

\begin{abstract}
Aim: The aim of the present study was to investigate the prevalence and distribution according to various arch length conditions of lingually erupted mandibular permanent incisors and the effect of space condition on this phenomenon with long-term follow-up of these teeth.

Methods and Materials: A total of 105 children aged 5-7.5 years with one or more permanent mandibular incisors erupting lingual to the corresponding primary incisors were included in the study. A space analysis was performed. The subjects were divided into six groups according to various space conditions. The subjects were followed for 2 years.

Results: In this study the prevalence of lingual eruption was $18.4 \%$. Eighty-three point seven percent of the subjects had either equivalent space, mild space excess, or a space deficiency (not more than $3 \mathrm{~mm}$ ). No relationship was found between groups and clinical outcomes ( $P>.05)$. Follow up of the main three groups (according to the space analysis results) revealed in the adequate space group $75 \%$ of the children lost their primary incisors without any intervention. Similar outcomes occurred in $85.7 \%$ and $57.14 \%$ of cases in the mild space excess and space deficiency groups, respectively. Considering the three main groups combined, equivalent space, mild space excess, and mild space deficiency, $70.45 \%$ of children lost their primary incisors without any intervention and $14.8 \%$ needed primary incisor extraction (17.4\%, $14.3 \%$, and $10.7 \%$ in each group, respectively).
\end{abstract}

(C) Seer Publishing 
Conclusion: There was no predisposition toward a space deficiency or excess found in these subjects so the wait-and-see policy versus early extraction can be considered for lingually erupting permanent mandibular incisors.

Clinical Significance: Lingual eruption of mandibular incisors is a common clinical problem in the early mixed dentition period that is a source of discomfort for parents of patients with this condition. The present study provides a unique insight into the prevalence of the problem as well as the clinical decisions such as favoring retention of primary incisors as long as possible, the extraction of these primary teeth, placement of lower lingual holding arches, or referral for comprehensive orthodontic treatment. These types of data should not be regarded as standards but as illustrative of several key factors that are still not well-understood or which are still ignored.

Keywords: Lingual eruption, permanent mandibular incisors, orthodontic treatment

Citation: Aminabadi NA, Farahani RMZ, Sohrabi A, Pouralibaba F. Lingual Eruption of Mandibular Permanent Incisors: A Space Correlated Phenomenon? J Contemp Dent Pract 2009 January; (10)1:025-032.

\section{Introduction}

Eruption of the mandibular permanent incisors lingual to retained primary incisors is often a source of concern for parents. Gellin and Haley ${ }^{1}$ emphasized anxiety is created when the parents discover a double row of teeth and suggested monitoring the condition without extraction is acceptable under certain conditions. McDonald ${ }^{2}$ suggested there are no significant contradictions to early removal of the offending primary incisors even in spaced dentitions. Extracting the primary teeth that interfere with the eruption of permanent teeth is one of the treatment choices to resolve lower anterior crowding during the mixed dentition period. ${ }^{3}$ However, parents' feelings should not be ignored in this decision-making. ${ }^{2}$

Indeed, there is a controversy surrounding extraction versus wait-and-see approaches, and this is often a source of confusion in the dental community. The condition is observed both in patients with an obvious arch length inadequacy and in those with a desirable amount of spacing of the primary incisors. Although there may be insufficient room in the arch for the newly erupted permanent tooth, its position will improve over several months. McDonald ${ }^{2}$ suggests spontaneous correction of lingually erupted permanent incisors is likely to occur given enough time, particularly in cases where there is not severe crowding. Even when lingual eruption of mandibular permanent incisors is seen in a patient with mild arch length deficiency, a watchful waiting approach may be justified.
During the period of transition from primary to permanent dentition, minor incisor crowding is often present in normally developing dentitions. Such crowding is often seen after the eruption of the succedaneous mandibular incisors. With the eruption of the permanent mandibular lateral incisors, mild incisor crowding represents a normal stage of development ${ }^{3}$ and is resolved by a slight increase in intercanine width, labial positioning of the permanent incisors relative to primary incisors, and slight distal movement of the canines into the primate spaces. ${ }^{4,5}$ According to Proffit and Fields, ${ }^{4}$ there are three sources for this regaining space: ${ }^{4}$

- With normal growth, a slight increase in arch width occurs across the canines of about $2 \mathrm{~mm}$ of space gain due in part to a labially inclined path of canine eruption.

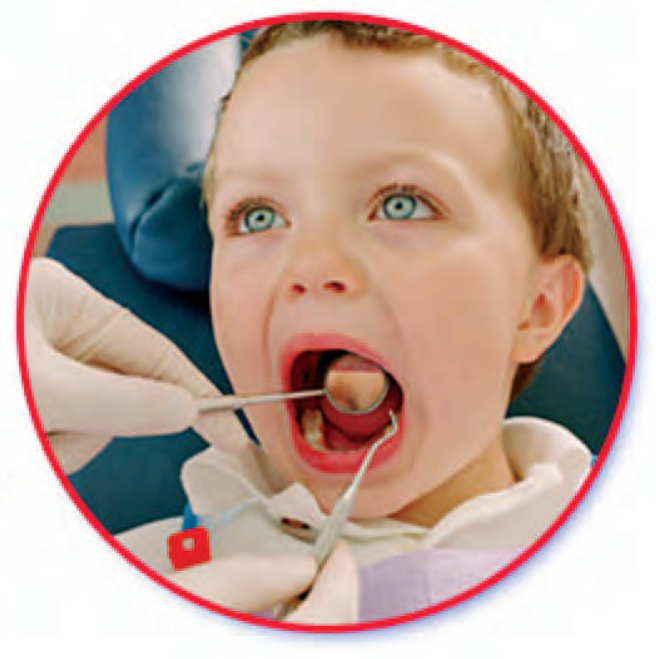


- As the succedaneous incisors replace their primary counterparts, they flare forward gaining 1 to $2 \mathrm{~mm}$ of arch length.

- In the mandibular arch the primate space is located posterior to the primary canines. Consequently, the permanent canines erupt in a more posterior position than their primary counterparts leaving the gained space of about $1 \mathrm{~mm}$ on each side available for the alignment of the incisors.

However, space analysis combined with evaluating the impact of compensating factors on dental arch status is the main approach by which overall space requirements for the lower arch can be determined during the mixed dentition phase. Overall space appraisal during the mixed dentition phase is highly indicative of future arch status. ${ }^{2}$

Few studies have investigated the prevalence and etiology of lingual eruption of permanent incisors.

This study evaluated the following:

- The prevalence of the lingual eruption of mandibular permanent incisors (erupting lingual to retained primary incisors)

- The effect of tooth size-arch length discrepancy on the condition

- The subsequent spontaneous correction of that condition based on space analysis over time.

\section{Methods and Materials}

A total of 582 children aged $5-7.5$ years with one or more erupting permanent mandibular incisors were included in this study. All subjects fulfilled the inclusion criteria:

- Early mixed-dentition stage (permanent mandibular incisors erupting)

- No congenitally missing permanent teeth

- No premature loss of primary teeth

- No damaged teeth due to caries (teeth with caries in the fissures only with intact walls were included)

- No previous orthodontic treatment

Short-term follow-up of all subjects was carried out for nearly one year until all permanent mandibular incisors (teeth \#71, 72, 81, and 82) were clinically visible $\left(A_{1}\right)$. Of those children, only 117 children (45 girls, 60 boys) were selected for the study $\left(A_{2}\right)$. During the follow up period, 12 children either discontinued recall visits or immigrated to other cities. Children were selected to continue in the study if they had one or more permanent mandibular incisors erupting immediately lingual to the corresponding primary incisors.

Alginate impressions were taken for 105 children (45 girls, 60 boys, aged 6-7.5 years). Casts were poured immediately using stone plaster. Arch perimeter measurements were made on the casts using electronic digital calipers (Digimatic Calipers, Mitutoyo, UK) based on methods used by Little, ${ }^{6}$ Sinclair and Little, ${ }^{7}$ and Bishara et al. ${ }^{8}$ Tooth materials were calculated using widths of permanent incisors on casts using the TanakaJohnston method for canines and premolars (mesiodistal widths of inferior permanent canine and premolars were estimated by summing $10.5 \mathrm{~mm}$ to the half of the sum of the lower four permanent incisors) ${ }^{9,10}$ The material was divided into six groups according to various tooth sizearch length discrepancy conditions: ${ }^{2}$

- The first group (space excess greater than 3 $\mathrm{mm}$ ) consisted of three children aged 6.3-6.7 years.

- The second group (space excess 0 to $3 \mathrm{~mm}$ ) consisted of 14 children aged 6-6.8 years.

- The third group (equivalency) consisted of 46 children aged 6-7.3 years.

- The fourth group (space deficiency less than $2 \mathrm{~mm}$ ) consisted of 28 children aged 6.2-7.5 years.

- The fifth group (moderate space deficiency between 3-6 mm) consisted of ten children aged 6.5-6.9 years.

- The sixth group (large space deficiency greater than $6 \mathrm{~mm}$ ) consisted of four children aged 6.5-7 years.

In the long-term follow-up phase of our study $\left(A_{3}\right)$, 88 children who had normal dental and skeletal occlusion were selected from the 105 children enrolled who had lingual eruption of permanent mandibular incisors. All subjects satisfied the following criteria:

- Normal dental arch space (belonging to the second, third, or fourth groups)

- Class I molar and canine interdigitation or flush terminal or mesial step terminal plane molar relationship

- Normal anterior and posterior overjet

- Normal anterior overbite

The mean age at $A_{1}$ phase was 5.6 years (range 5-7.4 years), and nearly one year later, mean age was 6.5 years (range 6-7.5 years) at $A_{2}$ 
phase when records were taken. Children were monitored according to Gellin and Haley, ${ }^{1}$ up to 8.5 years of age. If labial migration of the permanent mandibular incisors had not occurred by 8.5 years, the corresponding primary incisors were removed. If above-mentioned situations were accompanied by a mild crowding, the corresponding primary incisors were removed and a lower lingual arch holding arch was placed before extractions by 8.5 years old.

Dental visits and examinations were conducted in the Department of Pediatric Dentistry of the Tabriz School of Dentistry by an experienced pedodontist. The project was approved by the Committee for Ethics in Research on Humans of the Tabriz University of Medical Sciences.

\section{Statistical Analysis}

Descriptive statistics (prevalence and percentages) were calculated for the studied groups. Fisher's exact test was used to explore the effect of space condition on categorical values of clinical outcome with a p-value less than 0.05 considered statistically significant.

\section{Results}

\section{Measurement Error}

Ten randomly selected casts were re-measured and the method error calculated according to Houston. ${ }^{11}$ Houston's coefficient of reliability ranged from $96.3 \%$ for the arch perimeters to $92.1 \%$ for the tooth material measurements. A kappa test was applied to test intra examiner reliability for the space analysis. Ten children were examined on two occasions separated by one week. A Kappa value equal to 0.9 was achieved.

\section{Findings}

Frequency and percentage of lingual eruption cases in the total sample are presented in
Table 1. In this study the prevalence of lingual eruption was $18.4 \%$.

Distribution of lingual eruption cases in six different groups of space analysis is presented in Table 2.

As shown, when space analyses were done for lingual eruption cases, nearly half of children $(43.8 \%)$ had adequate space for accommodating successor teeth in the lower arch. When mild space excess and deficiency (not more than $3 \mathrm{~mm}$ ) pooled in the equivalent group, the percentile reached $83.7 \%$. Large space excess and deficiency only were seen in $2.8 \%$ and $3.8 \%$, respectively.

Table 3 shows distribution and percent of children in each group based on the clinical outcome at $A_{3}$.

The results of the statistical analyses are summarized in Table 4. As shown, all results were statistically not significant ( $p$-values $>.05$ ) indicating no relationship between space groups and clinical outcomes.

When the main three groups of children (according to space analysis results) were seen at follow-up $\mathrm{A}_{3}$, in the adequate space group, three fourths $(70.45 \%)$ of children lost their primary incisors without any intervention and $85.7 \%$ and $57.14 \%$ of children in the mild space excess and the space deficiency groups, respectively. Additionally, of the three main groups (equivalency, mild space excess, and mild space deficiency), $70.45 \%$ of children lost their primary incisors without any intervention and $14.8 \%$ needed primary incisor extraction $(17.4 \%, 14.3 \%$, and $10.7 \%$ of each group, respectively).

\section{Discussion}

The aim of the present study was to investigate the prevalence, distribution according to various

Table 1. Frequency of lingual eruption in the total sample $\left(A_{1}\right)$.

\begin{tabular}{|l|c|c|}
\hline \multicolumn{1}{|c|}{ Eruption Pattern } & N & Frequency (\%) \\
\hline Lingual eruption & 105 & 18.4 \\
\hline Non-lingual eruption & 465 & 81.6 \\
\hline Total & 570 & 100.0 \\
\hline
\end{tabular}


Table 2. Distribution of space analysis groups in lingual eruption cases $\left(A_{2}\right)$.

\begin{tabular}{|l|c|c|}
\hline \multicolumn{1}{|c|}{ Space Analysis Group } & N & Frequency (\%) \\
\hline Large space excess & 3 & 2.8 \\
\hline Space excess & 14 & 13.3 \\
\hline Equivalency & 46 & 43.8 \\
\hline Deficiency & 28 & 26.6 \\
\hline Moderate deficiency & 10 & 9.5 \\
\hline Large deficiency & 4 & 3.8 \\
\hline Total & 105 & 100 \\
\hline
\end{tabular}

Table 3. Distribution of space analysis groups according to clinical outcomes.

\begin{tabular}{|l|c|c|c|c|c|c|c|c|}
\hline \multirow{2}{*}{ Clinical Disposition } & \multicolumn{2}{|c|}{ Space excess } & \multicolumn{2}{|c|}{ Equivalency } & \multicolumn{2}{c|}{ Deficiency } & \multicolumn{2}{c|}{ Total } \\
\cline { 2 - 8 } & No. & Percent & No. & Percent & No. & Percent & No. & Percent \\
\hline Waiting, self-correction & 12 & 85.7 & 34 & 73.9 & 16 & 57.14 & 62 & 70.45 \\
\hline Primary incisor extraction & 2 & 14.3 & 8 & 17.4 & 3 & 10.7 & 13 & 14.8 \\
\hline Lower lingual holding arch & 0 & 0 & 3 & 6.5 & 6 & 21.4 & 9 & 10.2 \\
\hline Orthodontic intervention & 0 & 0 & 1 & 2.1 & 3 & 10.7 & 4 & 4.5 \\
\hline Total & 14 & 100 & 46 & 100 & 28 & 100 & 88 & 100 \\
\hline
\end{tabular}

Table 4. Calculated p-values for each of comparisons (one-sided).

\begin{tabular}{|l|c|c|c|}
\hline \multirow{2}{*}{\multicolumn{1}{|c|}{ Clinical outcome }} & \multicolumn{2}{|c|}{ Space Analysis Groups } \\
\cline { 2 - 4 } & $\begin{array}{c}\text { Space excess vs. } \\
\text { Equivalency }\end{array}$ & $\begin{array}{c}\text { Space excess vs. } \\
\text { Deficiency }\end{array}$ & $\begin{array}{c}\text { Equivalency vs. } \\
\text { Deficiency }\end{array}$ \\
\hline Waiting, self-correction & 0.300 & 0.063 & 0.108 \\
\hline Primary incisor extraction & 0.574 & 0.547 & 0.335 \\
\hline Lower lingual holding arch & 0.444 & 0.072 & 0.064 \\
\hline Orthodontic intervention & 0.767 & 0.285 & 0.149 \\
\hline
\end{tabular}




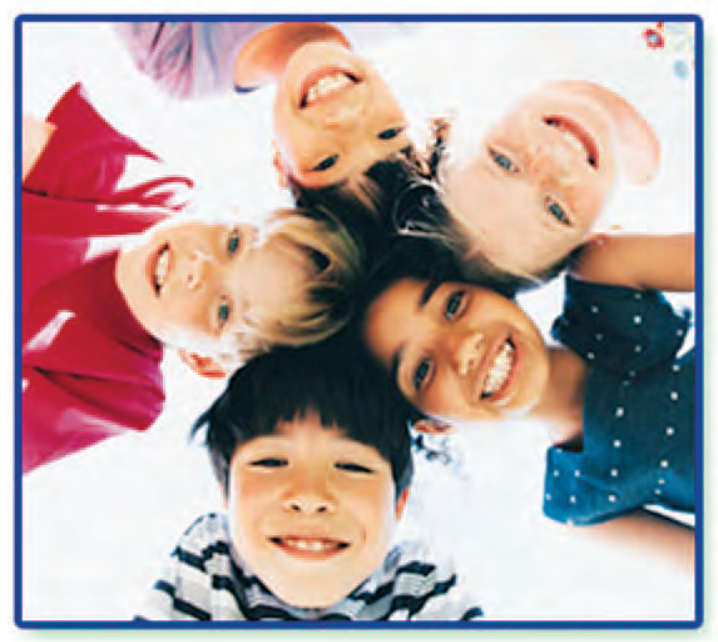

space conditions, the effect of space condition, and long-term follow up of the lingually erupting mandibular permanent incisors.

In this study the prevalence of lingually erupting mandibular permanent incisors was calculated as $18.4 \%$. This was higher than the $2.02 \%$ reported by Canoğlu et al. ${ }^{11}$ and the $10 \%$ of mandibular permanent incisors exhibited lingual eruptions reported by Garcia-Godoy et al. ${ }^{12}$ Several explanations exist for the observed relative disagreement. This diversity may be explained by racial and ethnic characteristics of the populations studied. Moreover, methodological aspects adopted in the studies, such as the criteria used in the sample selection, may also partially account for the observed difference. On the other hand, this difference can be attributed to differences in awareness and alertness of parents about the condition and by overall dental health of children. These data are based on clinic-based surveys, and the frequencies reflect the total sample of children who seek pedodontic treatment.

Interestingly, most of the lingually erupting permanent mandibular incisors belong to the children who had arches of equivalent space (43.8\%). Moreover, the prevalence of this condition was $26.6 \%$ and $13.3 \%$ in children who had deficient and excess space arches, respectively.

The findings of this study indicate lingual eruption of permanent mandibular incisors was not an arch space-related condition. This finding is supported by Gellin ${ }^{2}$ who emphasized the condition may be observed both in patients with an obvious arch length inadequacy and in those with a desirable amount of arch space. So it seems the etiology of this problem is not associated with space deficiency or excess problems. This finding is a predictable result because a previous study suggested the arch length, arch width, and intercanine measurements in these patients were not significantly different from the normal population. ${ }^{12}$

It can be postulated the etiology of the problem is related to either unfavorable eruption paths of these teeth, lingual positioning of tooth germs, or resorption resistance of primary roots due to partial ankylosis or other reasons.

In the third phase of this study, the long-term spontaneous mandibular incisor response in patients with normal and nearly normal arch length conditions (i.e., space excess less than $2 \mathrm{~mm}$, equivalency and space deficiencies less than $2 \mathrm{~mm}$ ) was assessed. Most believe dental arch space excess (1 to $2 \mathrm{~mm}$ ) is a relatively acceptable situation and, clinically, little intervention is usually required. On the other hand, space deficiencies less than $2 \mathrm{~mm}$ can be resolved during the transition from the primary to the permanent dental arch. ${ }^{2,5}$

During the observation period between the $A_{2}$ and $\mathrm{A}_{3}$ phases (nearly 2 years later), the majority of the children in the three groups (equivalency, mild space excess, and mild space deficiency) lost their primary incisors spontaneously and lingually erupted permanent incisors into normal positions without any intervention. Interestingly, this phenomenon was most prevalent in children who had excess space.

The scenario for mild space-deficient children differed slightly; in this group the observed spontaneous recovery was less than the normal space condition group (although it comprised more than half of the cases). Nonetheless, there was not a great need for primary incisor extraction in these cases and was less than the other two groups. The condition was spontaneously corrected through a 2-year period of waiting followed by placing a lingual holding arch in $21.4 \%$. Only $10.7 \%$ needed referral for comprehensive orthodontic treatment.

These findings are consistent with those reported by Gellin and McDonald ${ }^{2}$ who suggested 
spontaneous correction of lingually erupted permanent incisors is likely to occur given enough time even in cases with mild crowding (group 3 at $\mathrm{A}_{3}$ ). Thus, on the basis of the findings of this study, although intervention (primary incisor extraction and lower lingual holding arch) was a more frequent approach in the deficient space arches than in the other arch length conditions, the most frequent approach $(70.45 \%)$ was watchful waiting. Hence, lingual eruption of mandibular incisors does not require immediate intervention. Moreover, the results of this study revealed such an approach is not indicated in many cases and delaying treatment until later in dental development may be advisable.

Decisions favoring retention of primary incisors as long as possible, extraction of these primary teeth, placing lower lingual holding arches, or referring to comprehensive orthodontic treatment must be long-term decisions. Before any decision is made an accurate evaluation of arch length is necessary to confirm diagnosis. Thus, in children who have a normal dental and skeletal occlusion it may be reasonable to expect natural arch development and the action of a series of forces, influencing and guiding the permanent incisors into the more normal position with time.

The lingual eruption of permanent incisors is a self-correcting phenomenon and careful waitand-see policy is the best approach in any of these situations: (a) space excess, (b) space equivalency, (c) space deficiency less than 3 $\mathrm{mm}$, provided that no other dental and skeletal malocclusion exists.

\section{Conclusion}

The present study provided an insight into the state of the most prevalent conditions during teeth eruption, i.e., lingual eruption of permanent mandibular incisors. Lingual eruption of mandibular incisors is a common clinical problem in the early mixed dentition period that when encountered by parents is a source of discomfort. There is no predisposition toward a space deficiency or excess in these patients so the wait-and-see policy versus early extraction can be justified in these cases.

\section{Clinical Significance}

Lingual eruption of mandibular incisors is a common clinical problem in the early mixed dentition period that is a source of discomfort for parents of patients with this condition. The present study provides a unique insight into the prevalence of the problem as well as the clinical decisions such as favoring retention of primary incisors as long as possible, the extraction of these primary teeth, placement of lower lingual holding arches, or referral for comprehensive orthodontic treatment. These types of data should not be regarded as standards but as illustrative of several key factors that are still not well-understood or which are still ignored.

\section{References}

1. Gellin ME, Haley JV. Managing cases of overretention of mandibular primary incisors where their permanent successors erupt lingually. J Dent Children 1982: 118-122.

2. McDonald RE, Avery DR. Dentistry for the child and adolescent. 8th ed. Mosby, 2005, pp.184-6.

3. Yoshihara T, Matsumoto Y, Suzuki J, Sato N, Oguchi H. Effect of serial extraction alone on crowding: relationships between tooth width, arch length, and crowding. Am J Orthod Dentofacial Orthop.1999; 116:691-696.

4. Moorrees CFA, Chadha JM. Available space for the incisors during dental development- a growth study based on physiologic age. Angle Orthod. 1965; 35:12-22.

5. Proffit WR, Fields HW. Early stages of development. In: Contemporary orthodontics. 3rd ed. St. Louis: Mosby; 2000:86-91.

6. Little RM. The irregularity index: a quantitative score of mandibular anterior alignment. Am J Orthod. 1975; 68:554-563.

7. Sinclair PM, Little RM. Maturation of untreated normal occlusions. Am J Orthod. 1983; 83:114-123.

8. Bishara SE, Treder JE, Jakobsen JR. Facial and dental changes in adulthood. Am J Orthod Dentofacial Orthop. 1994; 106:175-186.

9. Tanaka MM, Johnston LE. The prediction of the size of unerupted canines and premolars in a contemporary orthodontic population. J Am Dent Assoc. 1974 Apr; 88:798-801. 
10. Irwin RD, Herold JS, Richardson A. Mixed dentition analysis: a review of methods and their accuracy. International J Paediatr Dent 1995; 5:137-142.

11. Houston WJB. The analysis of errors in orthodontic measurements. Am J Orthod 1983; 83:382-390.

12. Canoğlu H, Tekçiçek M, Turgut MD, Taner T. Lingual eruption of permanent mandibular incisors behind the deciduous teeth: Clinical Evaluation. Hacettepe Dişhekimliği Fakültesi Dergisi 2007; 31:89-94.

13. Garcia-Godoy F, Garcia-Godoy FM, Garcia-Godoy F. Caries experience in children with lingually erupted mandibular permanent incisors. J Pedod 1988; 12:176-8.

About the Authors

\section{Naser Asl Aminabadl, DDS, MSc}

Dr. Aminabadl is a Professor in the Department of Pedodontics of the School of Dentistry at the Tabriz University of Medical Sciences In Tabriz, East Azerbaljan, Iran. His research field Includes behavioral management of children, orthodontic correction of dentoskeletal anomalies, and root canal therapy of primary teeth.

e-mail: aslaminabadi@gmall.com

\section{Ramaln Mostofi Zadoh Farahanl, DDs}

Dr. Farahanl is a Research Assistant in the Department of Pedodontics of the School of Dentistry at Tabriz University of Medical Sclences in Tabriz, East Azerbaljan, Iran. His primary research Interests include wound healing and tissue regeneration in addition to his interest in the field of pediatric dentistry.

e-mall: rmostofi@gmall.com

\section{Aydin Sohrabl, DD8, MSe}

Dr. Sohrabl is an Assistant Professor in the Department of Orthodontics of the School of Dentistry in the School of Dentistry at Tabriz University of Medical Sclences in Tabriz, East Azerbaljan, Iran. His main research interest is in the orthodontic correction of dentofacial anomalies.

Firuz Pourallibaba, DDS, MSc

Dr. Pouralibaba is an Assistant Professor in the Department of Oral Diseases of the School of Dentistry at Tabriz University of Medical Sclences in Tabriz, East Azerbaljan, Iran. His main research interest is in oral diagnostic technologies. 